\title{
Light Curve Analysis of Long Period Pulsating Stars with the Wavelet Method: The semiregular star V Boo
}

\author{
K. Szatmáry, J. Gál and L. L. Kiss \\ Astron. Obs. and Dept. of Exp. Physics, JATE University, Dóm tér 9, \\ H-6720, Szeged, Hungary, e-mail: K.Szatmary@physx.u-szeged.hu
}

\begin{abstract}
We have collected long data series of some SR and Mira stars from data banks. In many cases the light curves show strong fluctuation or variation in the period, phase and amplitude. The detection of the significant changes of these parameters is important from the point of view of pulsation theory and stellar evolution. We present here the analysis of $V$ Boo. The used method is the Fourier and wavelet analysis in order to point out the modulations in the frequency and amplitude.
\end{abstract}

$\mathrm{V}$ Boo $=\mathrm{HD} 127335$ ( $\mathrm{V}=7.9 \mathrm{mag})$ is a SRa star of spectral type M6e similar to Mira stars, which was discovered by Dunér in 1884 . This star shows a light variation due to the pulsation with $P=258 \mathrm{~d}$. Percy et al. (1990) reported that the total amplitude decreased from 3 to 2 magnitudes between 1900 and 1975 .

Mattei et al. (1990) and Cannizzo et al. (1990) searched for chaotic behaviour in the AAVSO light curve of V Boo (1905-1965). They found that there is no evidence for deterministic chaos. They concluded that the light variation can be represented by a strictly periodic $(\mathrm{P}=259 \mathrm{~d})$ part and a random part whose maximal amplitude is one-half of that of the periodic part. Yanagita et al. (1993) used an AR model for the VSOLJ data, they determined $256.4 \mathrm{~d}$, $135.6 \mathrm{~d}$ and $888.9 \mathrm{~d}$ periods with $1.59,0.25$ and 0.17 mag amplitudes respectively. Evidence for chaos was not found.

Our analysis based on visual measurements collected by BAA, VSOLJ, AFOEV, AAVSO and HAA between JD 2420500 and 2449600 (1913-1994). The five data sets were merged and the magnitudes were averaged if there was more than one data point in a 10 day-long bin. The final light curve contains $\mathrm{N}=2648$ points (Fig. 1 left). The light change is Mira-like in the earlier part, and a strong amplitude decreasing can be seen later.

In order to study the amplitude and period variation we divided the whole data series to ten 3000 day-long segments. The amplitude spectra are in Fig. 1 (right). The next procedure was the determination of amplitude and phase for a fixed frequency component in each segment. The changes of these values are shown in Fig. 2. Assuming a linear amplitude variation the calculated rate is $d A / d t=4 \pm 1 \times 10^{-5} \mathrm{mag} / \mathrm{day}$ or $\Delta A / P=0.010 \pm 0.003 \mathrm{mag} / \mathrm{per}$. If this tendency continues the amplitude will be zero after the elapse of 15-20 years!

The wavelet transform was calculated for 50 frequencies and 100 time-shift (Szatmáry et al. 1994). In order to detect the effect of data distribution and gaps we have used a "whitening" method. The wavelet maps (frequency-timeamplitude) show the fine structure of the amplitude change. 

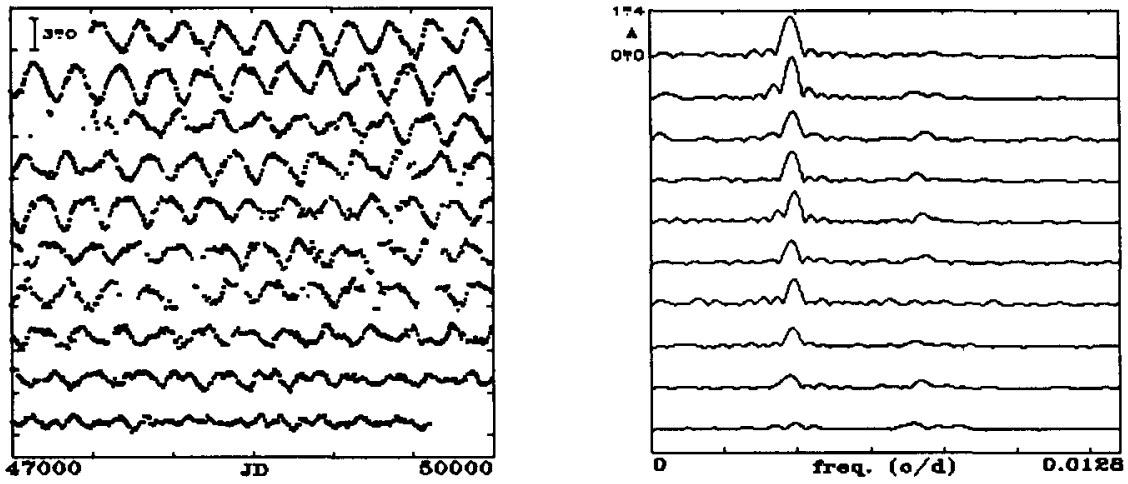

Figure 1. The light curves and the amplitude spectra of ten segments.
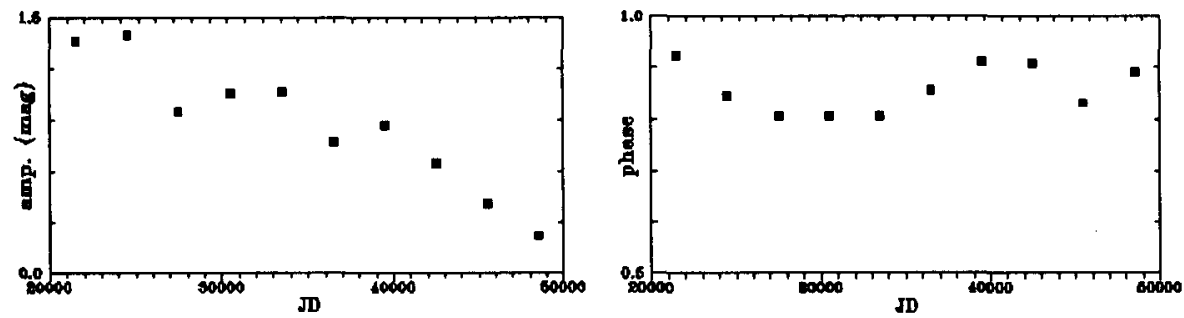

Figure 2. The amplitude and phase change of V Boo.

The O-C diagram, the plot of the instantaneous period and the CUSUM (Koen \& Lombard 1993) show no evidence for significant period variation.

We have tried to identify the pulsational modes based on the models of Fox $\&$ Wood (1982) and Ostlie \& Cox (1986). The possible modes for V Boo $\mathrm{P}=258$ and $137 \mathrm{~d}$ periods are the fundamental and first overtone. Using the relations for $P_{0}$ and $P_{1}$ the mass and the radius: $M=2.0 \pm 0.3 M_{\odot}$ and $R=290 \pm 20 R_{\odot}$.

The cause of the strong decline of the amplitude of $\mathrm{V}$ Boo is not clear yet. The estimated duration of the short-period Mira phase is $6 \times 10^{4} \mathrm{yr}$ $5 \times 10^{5} \mathrm{yr}$. The luminosity and the period is expected to increase in time based on evolutionary consideration.

The possible interpretation of the amplitude decreasing is that the fundamental pulsational mode quickly declines, while the strength of the first overtone is relatively stable. However the reason for this phenomenon is not known yet. Perhaps we see a star evolving from a short-period Mira state to a semiregular one. Perhaps by damping of the fundamental mode the first overtone will become the dominant mode. Might V Boo stop varying in the near future? Radial velocity measurements would be useful to answer these questions.

Acknowledgments. This analysis based on more than 22000 observations of some hundred amateur astronomers. We are grateful to the staff of AFOEV, VSOLJ and HAA for making their data available for us. This work was supported by the Hungarian OTKA grant No.T7522. 